\title{
Diagnosis and Management of Infected or Suspected Patients with Covid-19 a study of 48 Cases in the UK
}

\author{
Dan Jiang MMedSci, FBAcC, FATCM
}

Hallam Institute of TCM, 439 Glossop Road Sheffield S10 2PR UK.

*Corresponding Author: Dan Jiang, Hallam Institute of TCM, 439 Glossop Road Sheffield S10 2PR UK.

Received Date: 09 November 2021 | Accepted Date: 30 November 2021 | Published Date: 04 January 2022

Citation: D Jiang. (2022). Diagnosis and Management of Infected or Suspected Patients with Covid-19 A study of 48 Cases in the UK. Journal of Clinical and Laboratory Research. 5(1); DOI:10.31579/2768-0487/061

Copyright: (92022 Dan Jiang. This is an open-access article distributed under the terms of the Creative Commons Attribution License, which permits unrestricted use, distribution, and reproduction in any medium, provided the original author and source are credited.

\begin{abstract}
:
Covid-19 is a pandemic infective disease, which has been erupting throughout the whole world from 2020 to 2021. There have been at the time of reviewing more than a hundred million cases $(102,399,513)$ of infection, and more than 2 million deaths $(2,217,005)$ in more than 200 countries; this information is taken from the Covid-19 daily situation report issued on $1^{\text {st }}$ Feb 2021 by WHO. The pandemic was also declared as an outbreak of Public Health Emergency of International Concern on $30^{\text {th }}$ Jan 2020 by WHO. No effective treatment model has yet been confirmed by conventional western medicine, but some herbal treatments used in China can be recognized as having positive results in Covid-19 cases. This pandemic disaster has been severely damaging to quality of life, disturbing social communication, economic development and the progress of humanity.

Successful results were first reported from temporary hospitals (Fangcang Hospitals set up in arenas and exhibition spaces) in Wuhan, China, where more than $90 \%$ of patients treated with Chinese herbal medicine were prevented by transferring from the minor or milder stages of the disease to the severe or critical stage. In all of them the positive PCR became negative; herbs were involved in the rescue and treatment of severe and critical cases in ICU's in hospitals as well. It was an important factor in how Covid-19 was controlled so quickly in China.

The author recruited both confirmed and suspected Covid-19 patients through social media (WeChat, WhatsApp, Internet, message etc.) as a volunteer TCM consultant from March 2020, during the first Lockdown phase in UK. It was quickly realized that the appearance of the tongue provides special and significant information for identifying whether Covid-19 is present and shows its severity, from a TCM perspective, as the degree and type of dampness accumulated within the body. Herbal prescriptions were sent by post for patients' treatment according to their clinical findings, virus exposure history and tongue information (from photos). Similar positive effects were found as in China, and treatment reports and results are summarized here. The author believes there are many herbal medicines that can be effective in controlling the Sars-Cov-2 virus, and that prescribing the appropriate formula to match each individual case is the key point for TCM control of Covid-19. Chinese herbal medicine is the most effective current treatment method that can prevent cases in the minor and ordinary stages from progressing to the severe or critical stage, and so can play a significant role in the decrease and cure of Covid-19.
\end{abstract}

Key words: covid-19; corona virus; chinese herbal medicine; TCM

\section{Introduction:}

Covid-19 originally erupted in the central city of Wuhan in China Jan and Feb 2020, thousands of patients were infected within days, and all hospitals were rapidly overloaded with cases. As no effective medical treatment could be confirmed from conventional western medical experience, and there existed positive experience in the use of herbs in the successful treatment of SARS in 2003 [1, 2, 3], doctors began treating patients with herbal medicines in the temporary hospitals (Fangcang
Hospitals) which were reconstructed from arenas and exhibitions. More than $90 \%$ of patients were successfully treated after being given Chinese herbal medicine prescribed to prevent patients with Covid-19 in the minor or ordinary stages from progressing to the severe or critical stage, and herbal medicine was able to rescue many severe and critical cases in ICU's of hospitals as well. So, authorities in China issued national treatment guidelines [4] for Covid-19 introducing Chinese herbal medicine as the main therapy, to be given to patients as early as possible. This is a key point in explaining how control of Covid-19 was so quickly achieved in China. 

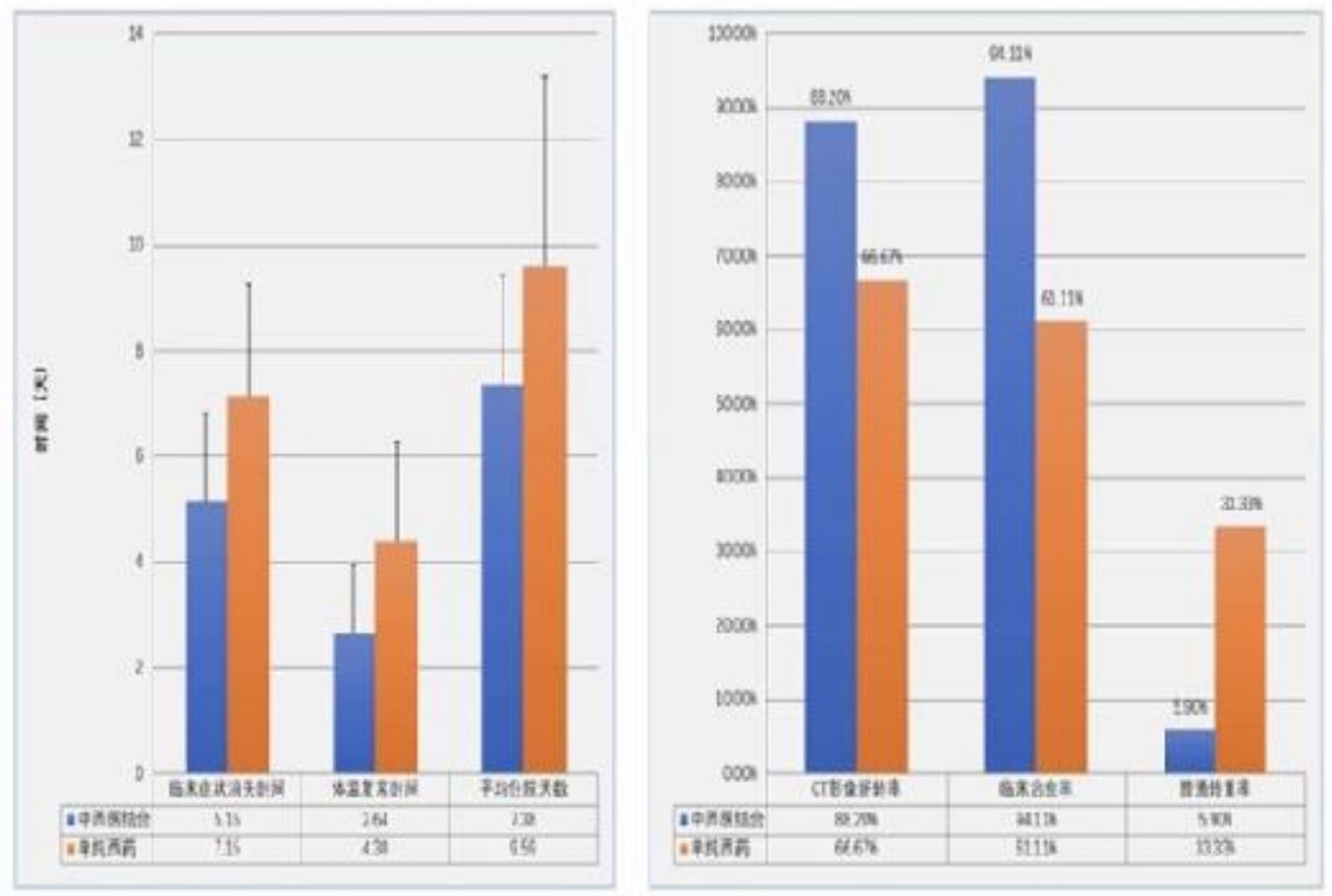

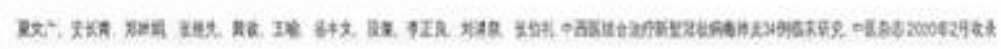

Note: on these charts the blue columns show the integrative approach; the orange columns show conventional medicine used alone.

An analysis made by TCM practitioner teams in China comparing results of Covid-19 treatment using TCM in an integrated way with conventional medicine, with conventional medicine used alone. Their results showed that the integrative approach decreased time to resolution of symptoms, to fever-clearance, and length of hospital stay; additionally the integrative approach increased the rates of clinical and radiological improvement, and also reduced the proportion of ordinary and minor cases which progressed to the severe form [5].

Covid-19 has been erupting and prevailing throughout the UK since the latter half of March 2020, when the Government introduced the first Lockdown phase. Although we have had to close complementary and alternative medicine clinics during this special time, the author still received many enquiries by remote forms of access, such as WeChat, WhatsApp, email, text messaging and so on daily, as a volunteer TCM consultant introduced by the overseas TCM forum and the Chinese Embassy in the UK (London). Many patients could not be admitted into hospitals or clinics for necessary medical treatment, although some nevertheless manifested quite typical and severe symptoms of Covid-19, and so had to be managed using the diagnostic methods of the TCM approach, and herbal treatments sent out to help them. A summary of the treatment method used is given here.

\section{The TCM treatment model used is outlined below:}

Necessary diagnostic procedures: clinical information is collected from patients, focusing on these aspects:

The history of exposure to Covid-19: Is there a history of contact with a Covid-19 infected person? If there is a history of possible contact, greater weight and attention is given to the patient's clinical symptoms.

Clinical symptoms:

Typical: fever, chills, cough, chest pain,

breathlessness, even

\section{dyspnoea;}

diarrhea,

Atypical: anorexia, loss of taste or smell, chest or upper back pain, red and

swollen toes,

blurred vision etc.

\section{Tongue appearance:}

The tongue body is used for determining how long the virus has been active within the body, and assessing the strength of the virus' resistance: for example, if the tongue is pale in color, this shows a sufferer who has been infected for a few ef days and who has had stomach weakness in the past. If the tongue is red in color, this shows a sufferer who may have been infected for more than a week, or has manifested higher fever, or has suffered greater stress in the past, or has a stronger constitution.

\section{Tongue coating:}

The presence of a greasy coating can indicate Covid-19 infection;

The color of the greasy coating, from white to yellow, identifies the severity of the heat inside the body;

The thickness of the greasy coating identifies the severity of the Covid19 infection. The thicker and more greasy the coating, the more severe the infection may be judged to be.

The tongue appearance provides unique and significant information from within the patient's body, following TCM's diagnostic approach.

Scientific diagnostic items: PCR, $\mathrm{SaO}_{2}$, Blood tests and CT scan. If patients have been admitted by the NHS, or accepted by their GP, these necessary examinations should have been carried out; some patients may 
possess a finger pulse oximeter for reading $\mathrm{SaO}_{2}$ by self-examination at home.

If anyone checks positive for at least two items amongst 1.1, 1.2 and 1.3, especially the greasy tongue coating which is a unique and significant sign for our diagnosis, we may assess them as infected or suspected patients with Covid-19, who should be given Chinese herbal medicine for treatment. While it is harder for us to obtain blood, PCR and CT scan results, the necessary examinations of 1.4 can confirm a diagnosis.

\section{Herbal medicine treatment:}

Because patients could not be seen face to face, we were unable to use acupuncture as a treatment, and Chinese herbal medicine was thus our only available treatment method. Some regulatory restrictions exist on the use of herbal medicine in UK, but we were able to use herbs and relevant formulae for treating Covid-19 as below:

\section{For patients with infected and suspected Covid-19 in the early stage:}

These patients display minor fever, shivering, minor cough, anorexia, loss of smell and taste, loose bowel movements, or dizziness, fatigue, general muscle aches etc. Pale or light red tongue with thin greasy coating.

Pattern of TCM: dampness accumulated in the lung;

Treatment principle: releasing excessive dampness.

The typical basic formula is Huoxiangzhengqi Decoction:

\begin{tabular}{|l|l|l|}
\hline \multicolumn{2}{|c|}{ Huoxiangzhengqi Decoction } & Doses \\
\hline Huoxiang & Herba Agastaches & 10 \\
\hline Houpo & Cortex Magnoliae Officinalis & 10 \\
\hline Fuling & Poria & 10 \\
\hline Zisu & Folium Perillae & 10 \\
\hline Dafupi & Pericarpium Arecae & 10 \\
\hline Jiangbanxia & Rhizoma Pinelliae & $10-15$ \\
\hline Chenpi & Pericarpium Citri Reticulatae & 10 \\
\hline Jiegeng & Radix Platycodi & 6 \\
\hline Baizhu & Rhizoma Atractylodis Macrocephalae & 10 \\
\hline Baizhi & Radix Angelicae Dahuricae & 6 \\
\hline Gancao & Radix Glycyrrhizae & 5 \\
\hline
\end{tabular}

The ingredients of Huoxiangzhengqi Decoction

With the above herbs as the main formula, we make modifications according to the particular characteristics of an individual case, and produce a prescription of $120-150 \mathrm{~g}$ of raw herbs which is decocted as herbal juice, half taken twice daily, one packet for each day; or we can also prepare them as herbal powders for drinking with warm water, twice daily.

\section{For patients infected with or suspected of Covid-19 in the ordinary stage:}

These patients manifest: fever and chills with a higher temperature, sometimes absent, chest tenderness or pain, breathlessness, more or less phlegm, dyspnea; or back-pain, painful, swollen toes or other symptoms. Red, or light red tongue with thick greasy, white-greasy, or yellow-greasy coating.

Pattern of TCM: excessive heat and dampness are accumulated in lungs; Treatment principle: expel excessive heat and dampness;

The typical basic formula is Qingfeipaidu Decoction: (National guideline in China)

\begin{tabular}{|l|l|l|l|l|l|}
\hline \multicolumn{7}{|c|}{ Qingfeipaidu Decoction } \\
\hline Xiangru(Mahuang*) & Herba Elsholtziae & $\mathbf{1 0}$ & Zhuling & Polyporus Umbellatus & 10 \\
\hline Guizhi & Ramulus Cinnamomi & $\mathbf{8}$ & $\begin{array}{l}\text { HuangQin } \\
(\text { Shiga } *)\end{array}$ & Radix Scutellariae & 10 \\
\hline Fuling & Poria & $\mathbf{1 0}$ & Kuandonghua & Flos Farfarae & 10 \\
\hline
\end{tabular}




\begin{tabular}{|l|l|l|l|l|l|}
\hline Shengjiang & $\begin{array}{l}\text { Rhizoma Zingiberis } \\
\text { Recens }\end{array}$ & $\mathbf{1 0}$ & Zhishi & Fructus Aurantii Immaturus & 10 \\
\hline Tinglizi(Xixin*) & Semen Descurainiae & $\mathbf{1 0}$ & Baizhu & $\begin{array}{l}\text { RhizomaAtractylodis } \\
\text { Macrocephalae }\end{array}$ & 10 \\
\hline Huoxiang & Herba Agastaches & $\mathbf{1 0}$ & Jiangbanxia & Rhizoma Pinelliae & Rhizoma Belamcandae \\
\hline Zexie & Rhizoma Alismatis & $\mathbf{1 0}$ & Shegan & Pericarpium Citri Reticulatae & 10 \\
\hline Chaihu & Radix Bupleuri & $\mathbf{6 - 1 0}$ & Chenpi & Rhizoma Dioscoreae & 10 \\
\hline Ziyuan & Radix Asteris & $\mathbf{1 0}$ & Shanyao & Radix Glycyrrhizae & $\mathbf{5}$ \\
\hline Xingren & Semen Armeniacae & $\mathbf{1 0}$ & Gancao & & 10 \\
\hline
\end{tabular}

The ingredients of Qingfeipaidu Decoction

* denotes herbs forbidden for use in the UK, so herbs with similar effects had to be substituted. We selected relevant herbs or formulae as appropriate for individual prescriptions, replacing forbidden herbs.

Using the above herbs as the main formula, we made modifications according to the particular demands of an individual case, producing a prescription of 120-150g of raw herbs a day decocted as herbal juice, half to be taken twice daily.

* There are many herbs in this prescription in the original formulation. We don't use all of these herbs in this formula in general in the UK, we select 12-15 herbs to make a suitable prescription according to the individual's condition, following the treatment principles of the formula.

\section{For people who need to be given double protection:}

People who may have minor clinical symptoms, such as feeling only some throat discomfort, shivering and are easily fatigued, but may be particularly vulnerable to Covid-19:

Medical staff or carers in daily or frequent contact with Covid-19 patients;

Family members, relatives and close friends of Covid-19 sufferers;

Seniors with chronic or severe respiratory or cardiovascular disease.

Treatment principle: promote protective Qi and release wind-dampness

The herbal tea bags of protective formula are as below:

\begin{tabular}{|l|l|l|}
\hline Huangqi & Radix Astragali & 10 \\
\hline Beishashen & Radix Glehniae & 10 \\
\hline Zhimu & Rhizoma Anemarrhenae & 10 \\
\hline Jinyinhua & Flos Lonicerae & 10 \\
\hline Lianqiao & Fructus Forsythiae & 10 \\
\hline Jiegeng & Radix Platycodi & 6 \\
\hline Cangzhu & Rhizoma Atractylodis & 10 \\
\hline
\end{tabular}

Ingredients of Protective formula $(g)$

Above herbal powders are made into tea bags, 1 tea bag once daily.

\section{Clinical summary:}

48 cases are here collected and summarized from patients with infected or suspected Covid-19 whom I have treated, and for whom I have kept complete clinical records:

\section{General state:}

Sex: Female: 27, Male: 21;

Age: eldest: 71; youngest: 11months;

Grouped by age: $\quad 0-24$ yrs. 6

$$
\begin{gathered}
25---45 \text { yrs. } 22 \\
46-64 \text { yrs. } 10 \\
65-70 \text { yrs. } 8 \\
>71 \text { yrs. } 2
\end{gathered}
$$

\section{Diagnosed state of Covid-19 infection:}

21 cases confirmed with a diagnosis of Covid-19 by a positive PCR (polymerase chain reaction) test;

27 suspected cases who manifested relevant symptoms, but without PCR test results*. 
*patients could not easily obtain a PCR test during the first lockdown phase, and some were also wary of being officially diagnosed with an infectious disease at that stage.

\section{State of treatment with Chinese herbal medicine:}

28 cases who took the decocted raw herbal prescriptive form;

5 cases who took the herbal powder prescriptive form;

37 cases who took the protective herbal tea bags.*

*Some cases manifesting only minor symptoms were treated with herbal tea bags only. Typical Covid-19 sufferers with fever and other more severe symptoms were given a dry herbal prescription for the first one to two weeks, and then given herbal tea bags once the acute symptoms had been controlled or had much decreased.

\section{Results:}

None of those taking herbal treatment deteriorated to become Covid-19 sufferers in the severe or critical-severe stages; all were stabilized or improved.

None of those taking only the protective herbal tea bag treatment went on to become full Covid-19 sufferers.

Those discharged from hospital still in a state of severe ill-health were able to recover fully with TCM treatment.

\section{Case studies:}

\section{Case 1:}

\section{Mrs. L, 38 year-old house-wife in London}

After her husband had been admitted into ICU in hospital, she became feverish, chilly, suffered persistent coughing for a week without help, temp. $38.3^{\circ} \mathrm{C}$, chest pain with breathlessness, anxiety, panic attacks, depression, nausea, anorexia and insomnia. She suffered from chronic gastritis, with a weak constitution. Pale tongue with white greasy coating.

Diagnosis: suspected Covid-19 in Cold-damp epidemic disease

Differentiation of syndrome: Cold-damp accumulated in the lungs with Spleen deficiency

Treating principle: warming cold and eliminating damp;

$$
\text { strengthening spleen and calming the mind. }
$$

Prescription: modified Huoxiangzhengqi combined with modified Wendan decoction $(\mathrm{g})$ :

Huoxiang 10, Houpo 10, Fuling 10, Banxia 10, Kouren 10, Sharen 10, Yiyiren 30, Huashi 15, Chenpi 10, Baizhu 10, Zhuru 10, Ganjiang 10, Gancao 5.

(Wendan provides needed mind-calming herbs, but strongly dampexpelling herbs were omitted because of the existing spleen deficiency and diarrhea)

Result: While taking herbs, she had some diarrhea, but was advised to keep taking the herbs until this stopped naturally; her tongue-coating cleared as well. She recovered after 2 weeks, but her husband died in hospital. She had a blood test which confirmed raised $\operatorname{IgE}$ and IgM levels after two weeks suffering Covid-19. A further two weeks of treatment with protective herbal tea bags was prescribed to complete her return to health, but he is told that her husband died in the hospital.

\section{Case1 tongue appearance:}

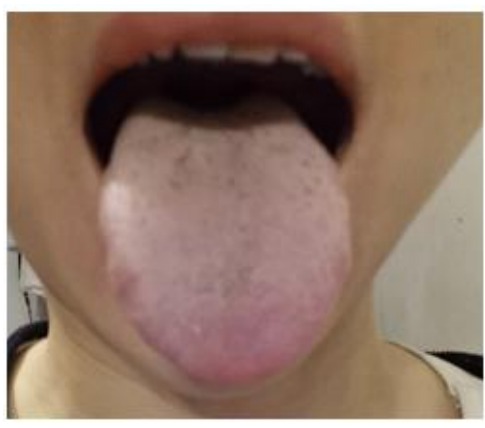

\section{Case2:}

Dr Z, 28; working as a junior doctor in UCLH

Temp. $39^{\circ}-40^{\circ} \mathrm{C}$ for 3 days with fatigue, tenderness and pain in the chest and breathlessness. She recognized she was suffering from Covid-19. She was worsening while taking only Paracetamol for treatment. Her worried mother sought help on her behalf from TCM.

Diagnosis: Covid-19;

TCM Differential Diagnosis by Syndrome: Damp \& Heat accumulated in the lungs

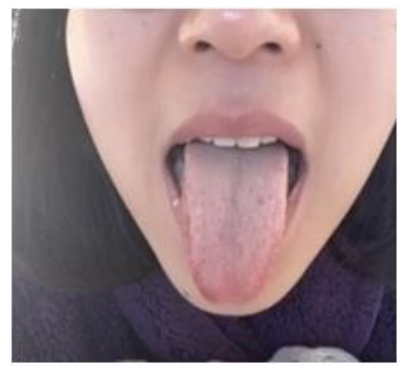

Prescription: Lianhuaqingwen decoction:

Jiyinhua 10, Lianqiao 10, Xiangru 10, Xingren 10, Shigao 30,

Guanzhong 10, Yuxingcao 10, Dahuang 10, Huoxiang 10, Daqingye 10,

Bohe 5, Gancao 5.

(Lianhuaqingwen is a formula for Covid-19 treatment introduced by the National Health Authority in China - see ref vi.)

Result: her temperature fell to $37.3^{\circ}-37.4^{\circ} \mathrm{C}$ over the next day, and she recovered sufficiently in a week to return to work.

\section{Case 3: Ms. B, 58 year-old clinical manager:}

She suffered a fever of $39^{\circ}-40^{\circ} \mathrm{C}$ with coughing and chest pain for a week, with breathlessness, dyspnea, constipation and insomnia. She did not want to go to hospital although she realized she might have Covid-19. Red tongue with a yellow cracked coating.

Diagnosis: a high suspicion of Covid-19 infection.

TCM Differentiation of Syndromes: Phlegm with damp and heat accumulated in the lungs; Obstruction in the large intestine

Prescription: Qingfeipaidu Decoction variation

Jiangbanxia 15, Chenpi 10, Huangqin 10, Gualou 10, Zhuru 10, Dahuang 10 (Later),

Laifuzi 10, Yuxingcao 10, Daqingye 10, Zhuling 10, Fuling 10, Zexie 10, Tinglizi 10,

Huoxiang 10, Gancao 5. 
Result: her temperature gradually decreased and she improved daily over a week. After she recovered, she showed less coating on her tongue. She was given a further two weeks of herbal tea bags to complete her recovery.

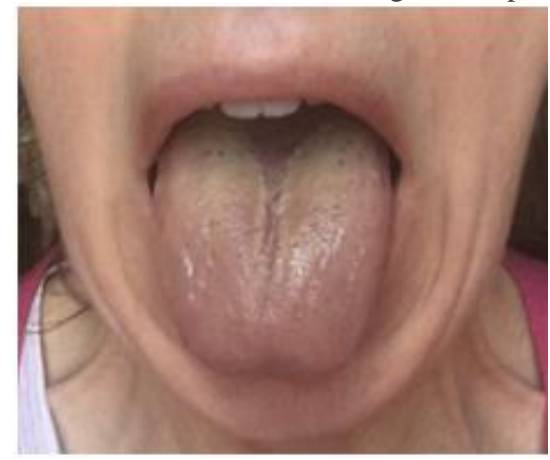

\section{Case 4: Mr. W 71, a visitor to London, a retired engineer} from Shanghai, China:

He and his wife came to London to visit their daughter's family, but he became infected in April and was admitted into ICU as his $\mathrm{SpO}_{2}$ fell to 83, with fever, severe breathlessness, chest pain and dyspnea. After having been discharged from ICU after one week his $\mathrm{SpO}_{2}$ was still low at around 89-91 with breathlessness, nightmares, open mouth while sleeping; fatigue, difficulty in walking, unable to move more than 10 mins in the flat or climb stairs. Tongue: red tongue with teeth marks at sides and white greasy coating

Diagnosis: Post-Covid-19 syndrome

TCM Differentiation of Syndromes: Qi and Yin deficiency with unclear phlegm-heat;

Prescription: Jiangbanxia 15, Chenpi 10, Fuling 10, Huangqin 10, Daqingye 10,

Chunalianzi 10,

$$
\text { Xuanshen 10, Beishashen 10, Maimendong 10, }
$$

\section{Gualou 10, Baihe 10, Taizishen 30, Gancao 5}

Result: The above herbal prescription was varied each week for a month, as he gradually recovered. His $\mathrm{SpO} 2$ increased to 95-96 in both wrists, he could walk down to and around his yard and return back up 4 flights of stairs to his flat without any palpitations, he experienced good sleep, regular bowel movements and a healthy color of complexion, lips and nails.

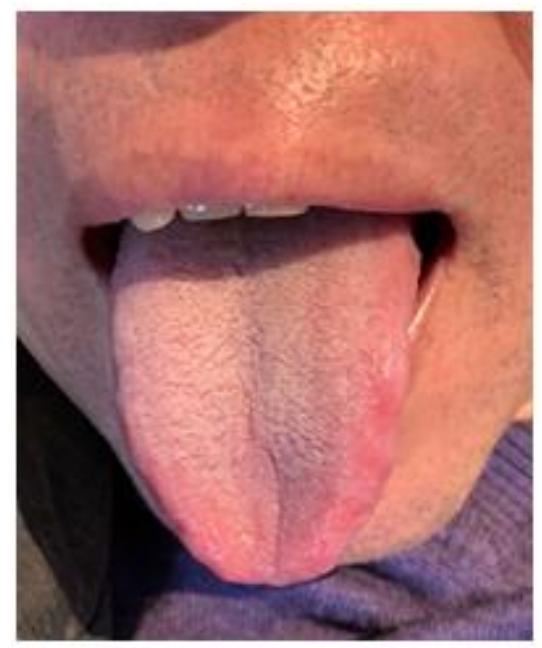

\section{Discussion:}

Chinese herbal medicine is an effective therapy to treat Covid-19. The $90 \%$ effectiveness rate which the TCM team claimed in the temporary hospitals in China was matched by our results, so their experience is confirmed as credible.

Covid-19 causes the accumulation of dampness in the lungs or damage to other organs from a TCM perspective, and we can gain specific and unique information from the tongue to confirm the presence and extent of this.

Chinese herbal medicine given at the early and middle stages can significantly help in preventing Covid-19 from worsening to the severe or critical stages;

Chinese herbal treatment possesses significant effectiveness in healing the post-syndromes of Covid-19. In particular herbs for reinforcing qi and nourishing yin can help patients recover completely, helping build general well-being and promoting

bio-circulation to prevent visceral fibrosis of the lung, heart or other organs

There may be many herbs which possess an anti-viral effect, so it is possible to

replace even some herbs which play a key part in the prescription. If the correct

replacement herbs are chosen, a new prescription can retain the expected effect.

Conclusion: Chinese herbal medicine is an effective therapy for the treatment of Covid-19.

\section{References:}

1. Zhao $\mathrm{CH}$, Guo $\mathrm{YB}, \mathrm{Wu} \mathrm{H}$, et al. (2003). "Clinical manifestation, treatment, and outcome of severe acute respiratory syndrome: analysis of 108 cases in Beijing"; Chinese Medical Journal. 83 (11):897-901.

2. Lau JT, Leung PC, Wong EL et al. (2005). "The use of an herbal formula by hospital care workers during the severe acute respiratory syndrome epidemic in Hong Kong to prevent severe acute respiratory syndrome transmission, relieve influenzarelated symptoms, and improve quality of life: a prospective cohort study." Journal of Alternative and Complementary Medicine. 11(1): 49-55.

3. Liu BY, He LY, Liang ZW, et al. (2005). "Effect of glucocorticoid with traditional Chinese medicine in severe acute aspiratory syndrome (SARS)". China Journal of Chinese Materia Medica Dec. 30(23):1874-1877.

4. (2020). National Health Commission \& National Administration of TCM, Diagnosis and Treatment Protocol for Novel Coronavirus Pneumonia - Trial Version 7thwas released on 3rd Mar 2020, Chinese Medical Journal. 133 (9), 1087-1095.

5. Xia WG, An CQ, Zhang BL. et al. (2020). "Clinical study of integrative medicine treatment of Novel corona pneumonia with 34 case studies". Journal of Chinese Medicine. 5; 1-5.

6. ZJ Zhang. (1993). translated by Xiwen Luo Treatise on Febrile Diseases Caused by Cold, New World Press in Beijing China.

7. JT Wu. (2017). Differentiation and Treatment of epidemic febrile diseases, China Publishing of Medical Science and Technology (Chinese) in Beijing China. 